\title{
Inhomogeneous nematic-isotropic phase transition of a thermotropic liquid crystal doped with iron oxide nanoparticles
}

\author{
Baeckkyoung Sung ${ }^{1,2,3^{*}}$, Huan Yan², Chanjoong Kim²,4, and Leon Abelmann ${ }^{1,5}$ \\ ${ }^{1}$ KIST Europe Forschungsgesellschaft mbH, 66123 Saarbrücken, Germany \\ ${ }^{2}$ Liquid Crystal Institute and Chemical Physics Interdisciplinary Program, Kent State University, Kent, $\mathrm{OH} 44242$, \\ USA \\ ${ }^{3}$ Division of Energy \& Environment Technology, University of Science \& Technology, 34113 Daejeon, Republic of \\ Korea \\ ${ }^{4}$ LG Chem R\&D Campus, 34122 Daejeon, Republic of Korea \\ ${ }^{5} \mathrm{MESA}^{+}$Institute for Nanotechnology, University of Twente, 7500 AE Enschede, The Netherlands
}

*Correspondence: sung@kist-europe.de

\begin{abstract}
We elucidate the local distribution and aggregated structure of iron oxide nanoparticles (IONPs; $6 \mathrm{~nm}$ in diameter) doped in the matrix of a nematic liquid crystal (LC), 4-cyano-4'pentylbiphenyl, utilizing in situ cryogenic transmission electron microscopy and polarized light microscopy. We show that tens of IONPs aggregate into a sphere-like morphology, and the aggregates combine into elongated clusters with a length of hundreds of $\mathrm{nm}$. With the IONP-doped LC matrix confined to a thin glass cell, we study the nematic-isotropic $(\mathrm{N}-\mathrm{I})$ phase transition, and suggest that local heterogeneity of LC textures as seen in polarized microscopy is caused by the existence of IONP aggregate clusters. These clusters act also as nuclei for the formation of isotropic domains upon heating.
\end{abstract}

Keywords: Thermotropic liquid crystal, nematic-isotropic phase transition, 4-cyano-4'pentylbiphenyl (5CB), iron oxide nanoparticle (IONP), cryogenic transmission electron microscopy (cryo-TEM), polarized light microscopy.

\section{Introduction}

Liquid crystals (LCs) hybridized with functional nanoparticles (NPs) have been extensively investigated as a means for improving the performances of electro-optic LC devices or as a scaffold platform to direct self-assembly of NPs. Small quantities of metallic NPs dispersed in LC media are known to enhance field responses [1,2], optical nonlinearity [3,4], birefringence [5], and photoluminescence [6,7]. Moreover, magnetic NP-doped nematic LC systems showed increased magneto-optic sensitivities under the presence of a static magnetic field $[8,9]$.

Colloidal NPs dispersed in LC matrices have been shown to create topological defects in the anisotropic host fluid, especially when spherically shaped NPs are suspended in the nematic medium [10]. In this case, the frustration of the nematic order tends to assemble the dispersed NPs into aggregates to minimize the elastic energy cost [11]. In equilibrium, the local deformation energy of the nematic matrix is related to the anchoring energy of the LC molecules on the NP surface and the effect of NP aggregation. In general, when the NP doping ratio increases, more NPs accumulate at the topological defects of the host LC [12]. Furthermore, the NP aggregation can also be driven by the phase transition of the LC media [11,13]. All these phenomena can be utilized to implement selfassembly templates for NP superstructures [14,15].

In this study, we investigate how the NPs organize within the LC scaffold, and what effect the presence of the NPs has on the nematic-isotropic $(\mathrm{N}-\mathrm{I})$ phase transition. Insight into the location and role of NPs inside LC media is crucial for the successful design of novel functional optical materials based on NP-doped LCs. The novelty of this work is two-fold: (1) We visualize directly how the 
spherical NPs are dispersed and clustered in the nematic matrix using cryogenic transmission electron microscopy (cryo-TEM). (2) We give evidence that the metal oxide NP aggregates can be nuclei for the local development of disordered domains during $\mathrm{N}-\mathrm{I}$ phase transition, under the condition of high doping ratio.

We have chosen thermotropic 4-cyano-4'-pentylbiphenyl (5CB) as the host LC material because it is one of the most studied nematic LC systems. In addition, bulk 5CB exhibits a well-defined $\mathrm{N}-\mathrm{I}$ phase transition behavior at around $35^{\circ} \mathrm{C}[16,17]$. Iron oxide nanoparticles (IONPs) were used as the dopant NPs because they have attracted wide interest due to the low dimension $(<10 \mathrm{~nm}$ in diameter) and thermal conductivity $[18,19]$. The localization and organization of the IONPs inside 5CB were imaged in situ by cryo-TEM. Polarized light microscopy was used to study the effect of dopant IONPs on the $\mathrm{N}-\mathrm{I}$ phase transition of host $5 \mathrm{CB}$ in real time under temperature control.

\section{Materials and Methods}

\subsection{Synthesis of Iron Oxide Nanoparticles (IONPs)}

IONPs were synthesized according to the protocol reported by Yan et al. [19]. To obtain a ferrite precipitate, $0.903 \mathrm{~g} \mathrm{FeCl}_{3} \cdot \mathrm{H}_{2} \mathrm{O}$ and $3.046 \mathrm{~g}$ sodium oleate were dissolved in a mixture solvent of 25 $\mathrm{mL}$ ethanol, $20 \mathrm{~mL}$ deionized water, and $45 \mathrm{~mL}$ hexane, followed by refluxing for $4 \mathrm{~h}$ at $62{ }^{\circ} \mathrm{C}$. The dispersion was rinsed with deionized water 3 times by extraction. Next, dried iron oleate complex and $0.6 \mathrm{~g}$ oleic acid were dissolved in $20 \mathrm{ml}$ dioctyl ether at $70{ }^{\circ} \mathrm{C}$. The mixture was heated to $290{ }^{\circ} \mathrm{C}$ for $1.5 \mathrm{~h}$. Iron oxide nanoparticles were collected by centrifugation at $6000 \mathrm{rpm}$ in ethanol. Then the particles were re-dispersed in hexane and extra ethanol was added, followed by centrifugation at $6000 \mathrm{rpm}$. This step was repeated 3 times to remove residual impurities. The IONPs were dispersed in $40 \mathrm{~mL}$ hexane with $100 \mu \mathrm{L}$ oleic acid and kept at $4{ }^{\circ} \mathrm{C}$, allowing the particle surface to be coated with oleic acid. The diameter of the coated IONPs was approximately $6 \mathrm{~nm}$ [19]. The concentration (number density) of the IONPs in the final suspension was $2 \times 10^{16} / \mathrm{mL}$.

\subsection{Preparation of IONP-Doped Nematic Liquid Crystal (LC) Cells}

In a test tube, $10 \mu \mathrm{L}$ or $33 \mu \mathrm{L}$ of the stock IONP dispersion was mixed with $100 \mu \mathrm{L}$ of the liquid crystal 4-cyano-4'-pentylbiphenyl (5CB) and left for 1-2 weeks at room temperature to allow slow evaporation of hexane, to finally resulted in the IONP concentration (number density) at $2 \times 10^{15} / \mathrm{mL}$ ("IONP-poor") and $7 \times 10^{15} / \mathrm{mL}$ ("IONP-rich"), respectively. Then the mixture was spun and vortexed repeatedly for 20 $\min$. A few $\mu \mathrm{L}$ of the mixture was administered on a slideglass and covered by a coverslip $(22 \times 30$ $\mathrm{mm}$ ). Before the LC cell preparation, all glass substrates were cleaned by sonication in $70 \%$ ethanol $\left(20 \mathrm{~min}, 50^{\circ} \mathrm{C}\right.$ ), rinsed with deionized water, and blow-dried with nitrogen gas. Some of the LC cells (shown in Fig. 1b, 1b') were fabricated with the glasses that were dip-coated with aqueous hexadecyltrimethylammonium bromide $(C T A B)$ to induce homeotropic alignment of $5 C B$ molecules [13]. [All the other optical microscopy images were taken with the samples in LC cells of non-treated clean glasses.] The edge boundary between the coverslip and slideglass was sealed with ultraviolet hardening epoxy glue. The closed LC glass cells created had a final thickness (i.e., the spacing between the two glass surfaces) of $10.5 \pm 0.8 \mu \mathrm{m}$, which was checked using differential interference contrast (DIC) microscopy (BX51, Olympus, Japan). 


\subsection{Cryogenic Transmission Electron Microscopy (Cryo-TEM)}

The cryo-TEM experiment was performed following protocols reported by Gao et al. [20] and Sung et al. [21]. Approximately $3 \mu \mathrm{L}$ of the 5CB-IONP mixture was deposited on a perforated carbon grid (Quantifoil R2/2, Jena, Germany). Before loading, the carbon-coated grid was treated by a plasma cleaner (Model 1020, Fischione Instruments, Export, PA, USA). By using a cryo-plunger (Vitrobot, $\mathrm{FEI}$, Hillsboro, OR, USA), the grid was blotted with a filter paper for $1 \mathrm{~s}\left(22{ }^{\circ} \mathrm{C}\right)$ and immediately plunged into liquid ethane. The frozen specimen was then transferred into a Gatan 626.DH cryoholder (Gatan, Warrendale, PA, USA) and observed in a TEM (Tecnai F20, FEI, Hillsboro, OR, USA) operated at $200 \mathrm{kV}$. The images were captured by a CCD camera (UltraScan $4 \mathrm{~K}$, Gatan, Warrendale, PA, USA) under low electron dose conditions at a maximum magnification of $43000 \times$.

\subsection{Polarizing Microscopy with Temperature Control}

The cell containing the 5CB-IONP mixture was placed in a homemade temperature-controlled stage and observed with an optical microscope (BX51, Olympus, Japan) equipped with a CCD camera (B1943, Imperx, USA) and a 40x objective (NA 0.75, UPLANFL) for polarized light microscopy. Additionally, a Laborlux 12 polarized light microscope (Leitz, Germany) equipped with a D5600 DSLR camera (Nikon, Japan) was used to observe the liquid-crystalline textures between crossed polarizers at low-magnification in RGB mode. This temperature-controlled imaging system was designed to observe the nematic-isotropic phase transition behaviors of the mixture during the temperature change in real time and high detail. The heating cycles were carried out at temperatures between 22 ${ }^{\circ} \mathrm{C}$ and $42{ }^{\circ} \mathrm{C}$ at a rate of $0.3-0.4{ }^{\circ} \mathrm{C} / \mathrm{min}$.

\subsection{Image Processing and Data Analysis}

All the optical and electron micrographs, including individual images and time-lapsed image series stacks, were numerically treated and analyzed with ImageJ software (downloaded from www.imagej.nih.gov/ij/NIH, Bethesda, MD, USA). For the measurements of the IONP diameter and inter-IONP spacing, respectively, radial integration and line profile functions were applied on the images. The software OriginPro 2019b (OriginLab, Northampton, MA, USA) was used for curve fitting and data statistics.

\section{$3 \quad$ Results and Discussion}

\subsection{Aggregation of IONPs in the Nematic Host Matrix}

We observed the effect of oleic acid-coated IONPs on the 5CB liquid crystal both in polarized light microscopy and cryo-TEM. For polarized light microscopy, pure and doped liquid crystals were confined to thin glass cells. In the nematic phase of host 5CB, typical nematic textures, such as Schlieren patterns, could be seen (Fig 1a). These patterns are particularly prominent when the doping concentration was small (Figs. 1c, 2a, and 3a). These observations are consistent with previous investigations on the electro-optic properties of nematic LC devices doped with metallic NPS at low concentrations. There it was found that the dopants contributed to the enhancement of the field-response with little effect on the global nematic order of the LC cells [2].

Nevertheless, even at low doping ratio homeotropically aligned LC specimens show sparsely distributed NP aggregates that induced local deformation of the orientational order in the nematic 
matrix (Fig. 1b, 1b'). As we increased the IONP doping ratio, we observed more prominent appearance of these inhomogeneous textures (i.e., individual and/or segregated granules), predominantly at the defects of the nematic phase (Fig. 1c, 1d).

The resolution of polarized optical microscopy is insufficient to image the individual nanoparticles. Therefore we observed the doped liquid crystal by cryo-TEM at magnification up to 43000x. At this high magnification, individual IONPs could be clearly identified as electron-dense points (Fig. 2b-e). The IONP diameter was measured for more than 100 particles. The average diameter was $5.8 \pm 0.1$ $\mathrm{nm}$ (standard error). The distribution of the IONP diameters, which has a standard deviation of 1.2 $\mathrm{nm}$, was fitted to a Gaussian curve, resulting in an average diameter of $5.7 \pm 1.1 \mathrm{~nm}$ (standard deviation).

The IONPs were closely-packed in sphere-like aggregates (Fig. 2c-e), of which the average diameter was $34.7 \pm 0.9 \mathrm{~nm}$ with a standard deviation of $9 \mathrm{~nm}$. The inter-particle (center-to-center) distance was $7.72 \pm 0.08 \mathrm{~nm}$ with a standard deviation of $1.0 \mathrm{~nm}$. From the difference between the IONP diameter and inter-particle distance, we conclude that the inter-particle surface-to-surface distance is $1.9 \pm 0.2$ $\mathrm{nm}$. This surface-to-surface distance corresponds to the length of oleic acids, which is approximately $2 \mathrm{~nm}$ [22]. We assume that the IONPs in the aggregates are closely packed, sterically spaced by a layer of overlapping oleic acid chains. Assuming a perfectly spherical aggregate consisting of closelypacked IONPs with a density of 0.74 , the most occurring number of IONPs per aggregate is $36 \pm 6$. The distribution of the number of particles per aggregate is highly asymmetric, the median is 67 and $80 \%$ of the aggregates contained between 18 and 185 particles (10-90 percentile).

Both polarized light and transmission electron microscopy indicate that the IONPs aggregate in close packed structures. The driving force for the close packing of IONPs can be attributed to the repulsion into topological defects in the nematic order of 5CB. The topological defect-based aggregation of 5-6 $\mathrm{nm}$-sized NPs is a Brownian fluctuation-driven process occurring at equilibria [11]. It is suggested that this aggregation mechanism results in a fractal-like clustering via slow assembly of smaller aggregates [11]. Our experiments imply that the closely packed sphere-like aggregates initially formed locally, and then migrated to the defect sites to be clustered with other sphere-like aggregates.

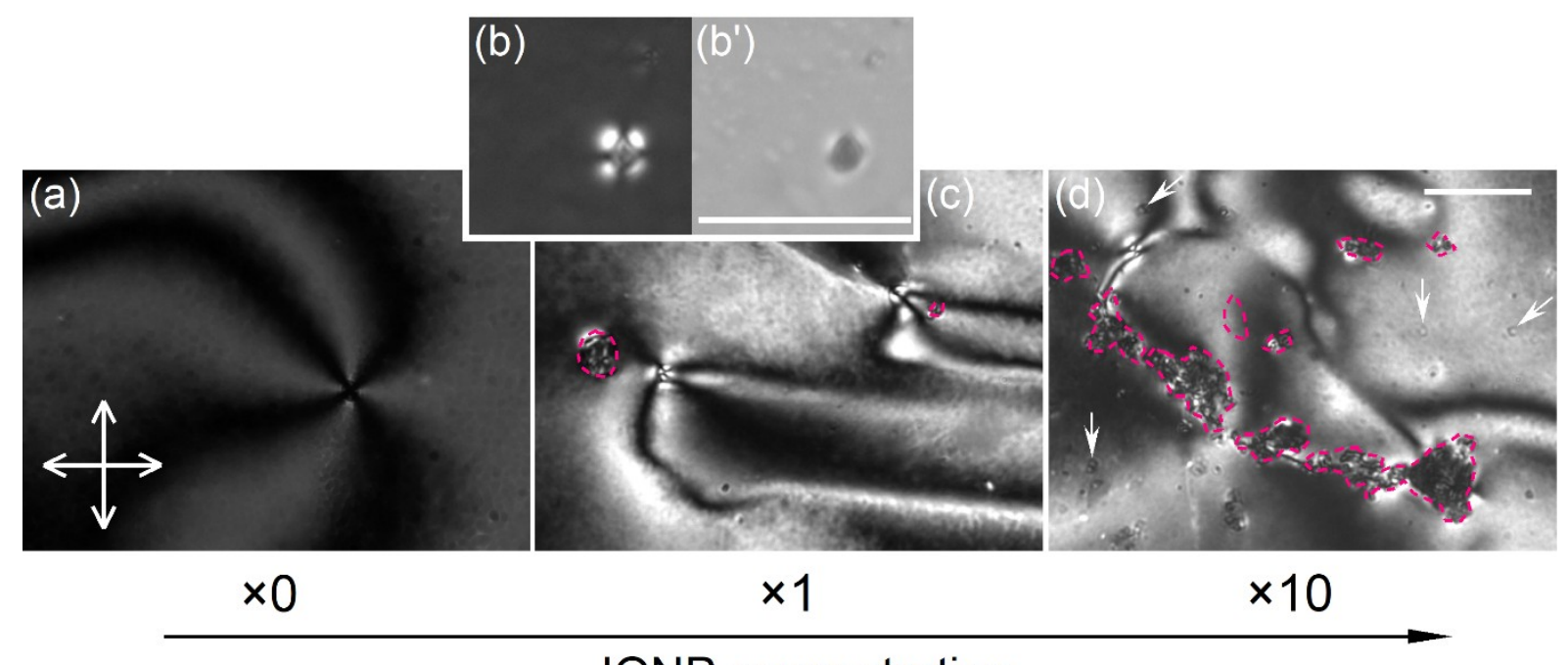

IONP concentration

Fig. 1. Polarized light microscopy images of the nematic liquid crystal mixtures: (a) pure 5CB. (b-d) 5CB doped with iron oxide nanoparticles (IONPs). (b-c) 5 CB at a low doping ratio of IONP $\left(2 \times 10^{15} / \mathrm{mL}\right.$ ) in the LC cells with (b) CTAB-coated glasses and (c) non-treated glasses. In (b,b'), nematic 5CB molecules are in homeotropic alignment. The images (in the same field of view) show two aggregates of IONP and were taken between (b) 
crossed polarizers, and (b') opened polarizers. (d) 5CB doped with highest amount of IONPs $\left(2 \times 10^{16} / \mathrm{mL}\right)$. Individual (arrows) and localized (dashed lines) granules (i.e., IONP aggregates) are present in the nematic matrices. Scale bars, $50 \mu \mathrm{m}$.
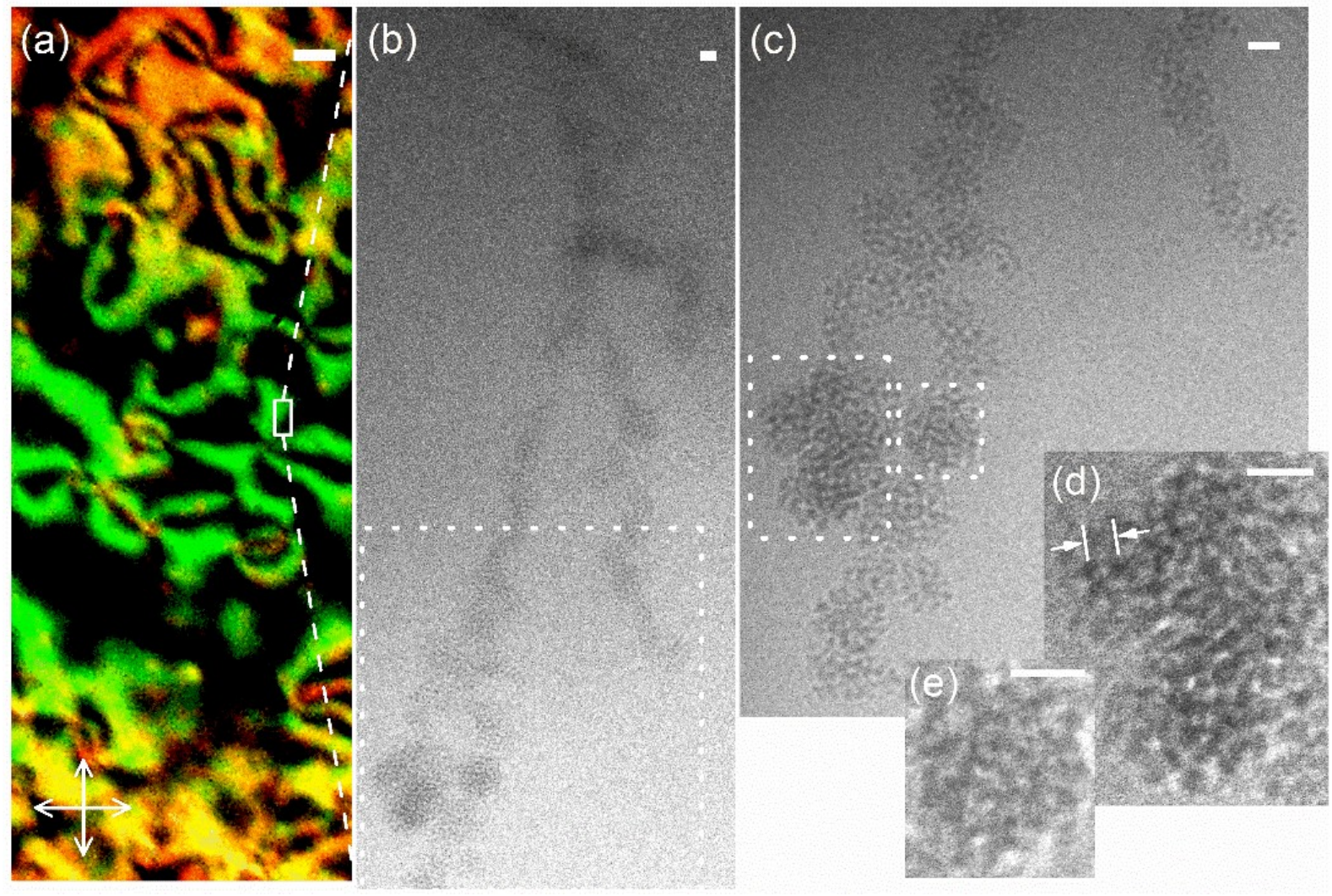

Fig. 2. Structures of aggregated IONPs that are doped in the nematic $5 \mathrm{CB}$ media. (a) Low-magnification image of the 5 CB-IONP mixture (IONP $2 \times 10^{15} / \mathrm{mL}$ ) between crossed polarizers. Scale bar, $50 \mu \mathrm{m}$. (b) Cryo-TEM image of the global morphology of the IONP aggregate clusters in the 5CB matrix. (c) Enlarged view of the dotted rectangle in (b). Each electron-dense point represents a single IONP. (d) Enlarged view of the left dotted rectangle in (c). A distance between two adjacent IONPs is indicated by arrows. (e) Enlarged view of the right dotted rectangle in (c), representing a "unit" sphere-like aggregate of the cluster shown in (b). (b-e) Scale bar, 20 $\mathrm{nm}$. 


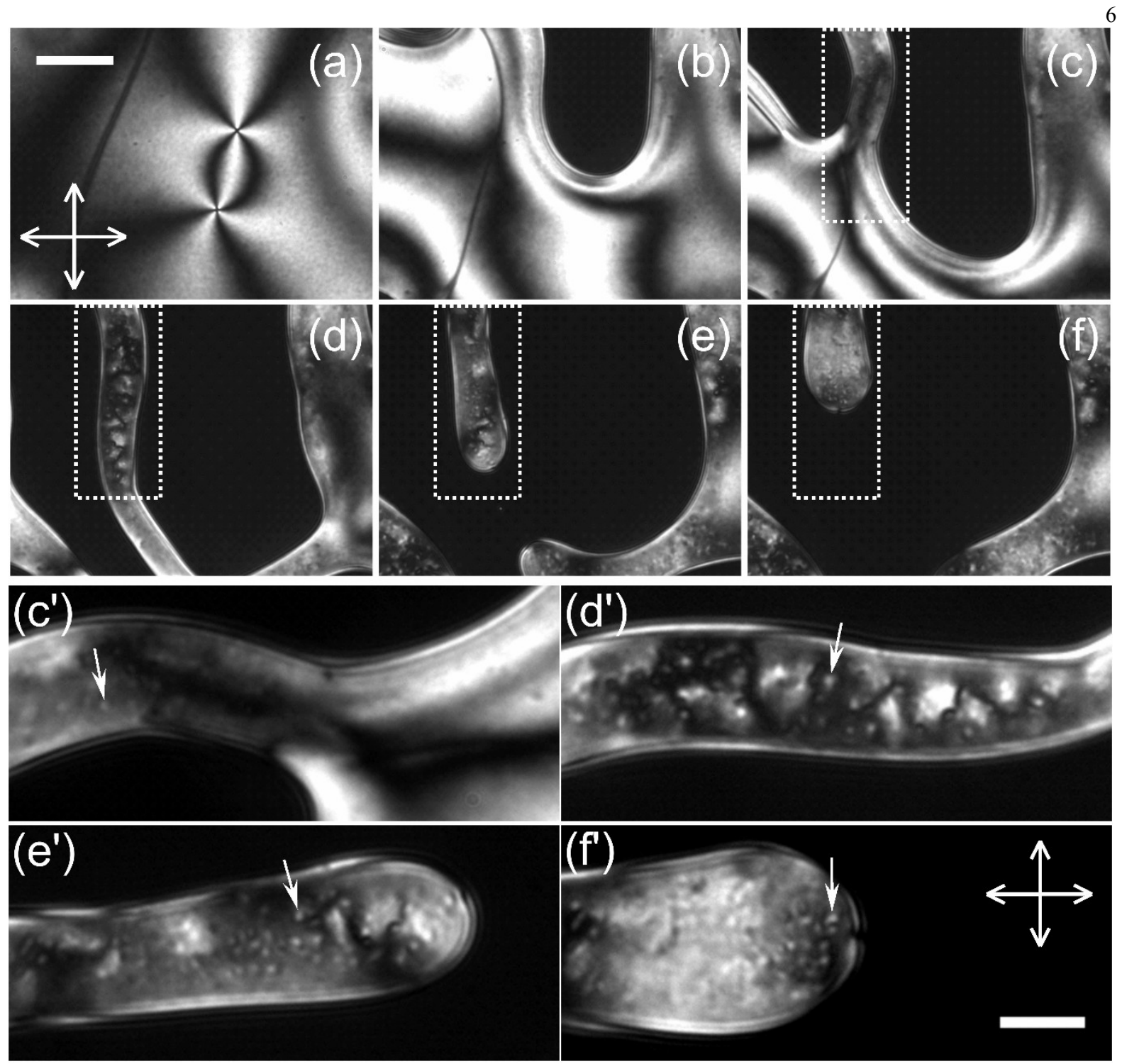

Fig. 3. Monitoring the nematic-isotropic (N-I) phase transition of the 5CB-IONP mixture (“IONP-poor", $2 \times 10^{15} / \mathrm{mL}$ ) by using polarizing microscopy. (a-f) Gradual melting of the mixture beginning from a complete nematic phase. The time interval between each adjacent image is 1-2 s. Scale bar, $50 \mu \mathrm{m}$. (c', d', e', f') Enlarged views of the dotted boxes in (c), (d), (e), and (f), respectively. The arrows indicate the granular textures appearing in the inhomogeneity during the phase transition. Scale bar, $20 \mu \mathrm{m}$.

\subsection{Optical Microscopy of 5CB-IONP Mixtures During Nematic-Isotropic Transition}

By slowly increasing the temperature, the nematic-isotropic $(\mathrm{N}-\mathrm{I})$ phase transition of IONP-doped nematic 5CB was monitored under a polarized light microscope (Fig. 3). As in Fig. 2a, when the doping quantity was small, a well-characterized nematic texture was observed under cross polarizers below the phase transition temperature (Fig. 3a). When the melting began, macroscopically separated nematic and isotropic domains coexisted. The isotropic domains became larger as the phase transition progressed (Fig. 3b-f). Interestingly, inhomogeneous patterns at the micrometer scale appeared in the nematic milieu (Fig. 3c-f). This inhomogeneous texture consisted of locally segregated and highly birefringent granules occupying the darker regions (Fig. 3c', d', e', f'). The 
granules were similar to those that appeared in the nematic matrix at the highest doping ratio in Fig. $1 \mathrm{~d}$, and the density of these granules increased as the transition approached the full isotropic phase. Because such local inhomogeneity was never observed during the usual $\mathrm{N}-\mathrm{I}$ phase transition of pure $5 \mathrm{CB}$, and taking into account the result shown in Fig. 1, we hypothesized that these granular textures (under crossed polarizers) could be directly related to the clusters of aggregated IONPs, which we observed in the cryo-TEM. The appearance of granules in the course of the N-I phase transition would be due to the increase of IONP concentration in the nematic domain: It has been reported that the dispersed NPs in 5CB tend to remain in the shrinking nematic region during the $\mathrm{N}-\mathrm{l}$ phase transition [23]. It is also well known that metallic NPs dispersed in nematic host 5CB can be led to aggregate because of the expulsion by the LC molecules to defect sites [12,23].

To verify this hypothesis, we repeated the same experiment with an increased concentration of dopant IONPs (Fig. 4). With a high amount of IONPs, the granular textures observed in Fig. 3c-f were dispersed throughout the nematic phase, together with large localized and segregated granules at the defect sites (Fig. 4a). The size of the granules was $2.03 \pm 0.05 \mu \mathrm{m}$, distributed with a standard deviation of $0.7 \mu \mathrm{m}$. As the $\mathrm{N}-\mathrm{I}$ phase transition progressed, microscale droplets were nucleated from the granules and grew with time finally to be dark isotropic domains (Fig. 4b-d). Simultaneously, the granules acted as the local centers for the $\mathrm{N}-\mathrm{I}$ phase transition where the isotropic domains formed earlier than the surroundings (Fig. 4b). Because it has been previously reported that the aggregates of metallic NPs in the isotropic host facilitate the local "crystallization" to nematic domains upon cooling $[23,24]$, we conclude that the granular textures in the crossed polarizers essentially correspond to the scattered light from the clusters of aggregated IONPs (shown in Fig. $2 \mathrm{~b}$ ) that were probably combined with locally disoriented $5 \mathrm{CB}$ molecules, which were in contact with the clusters. Because the size of the clusters is comparable to the wavelength of visible range, the clusters may cause considerable light scattering [25] and are clearly visible in optical microscopy. This may also be the reason why the detected granules have a minimum size of $0.7 \mu \mathrm{m}$ under polarized light microscopy, which is close to the limit of the optical resolution for our setup.

There was no prominent difference between the LC cells made of CTAB-coated glasses and nontreated glasses, in terms of the $\mathrm{N}-\mathrm{I}$ phase transition patterns. The $\mathrm{N}-\mathrm{I}$ transition temperature $\left(\mathrm{T}_{\mathrm{N}-\mathrm{I}}\right)$ of the "IONP-poor" and "IONP-rich" mixtures were measured to be $31.6 \pm 0.6{ }^{\circ} \mathrm{C}$ and $32.0 \pm 0.6{ }^{\circ} \mathrm{C}$, respectively, which had no significant difference with that of pure $5 \mathrm{CB}\left(32.5 \pm 0.6{ }^{\circ} \mathrm{C}\right)$ in the same type of $L C$ cell. The measured $T_{N-1}$ of pure $5 C B$ showed a full consistence to the previous reports by ParraVasquez et al. [26] $\left(\mathrm{T}_{\mathrm{N}-\mathrm{I}}=32 \pm 1{ }^{\circ} \mathrm{C}\right)$ and Rauch et al. [27] $\left(\mathrm{T}_{\mathrm{N}-1}=31.2{ }^{\circ} \mathrm{C}\right)$, where the comparable thickness of LC glass cells were used.

In previous works by Rodarte et al. [13] and Melton et al. [11], the "crystallization" (i.e., I-N transition) of NP-doped disordered 5 CB upon cooling was shown to repel the suspended NPs $(6 \mathrm{~nm}$-sized quantum dots) from the growing nematic domains to the shrinking isotropic domains. This phenomenon was assumed to be due to the entropic sorting of the colloidal NPs out of the ordered matrix of nematic 5CB. In our experiments, in contrast, the suspended metal oxide NPs with comparable size and shape preferentially remained in the contracting nematic domains during the melting (i.e., $\mathrm{N}-\mathrm{I}$ transition) of the ordered phase of $5 \mathrm{CB}$ upon heating. We speculate that the $\mathrm{I} / \mathrm{N}$ interfacial barrier might confine the colloidal NPs in the initially dispersed phase during the rapid propagation of the biphasic front. 

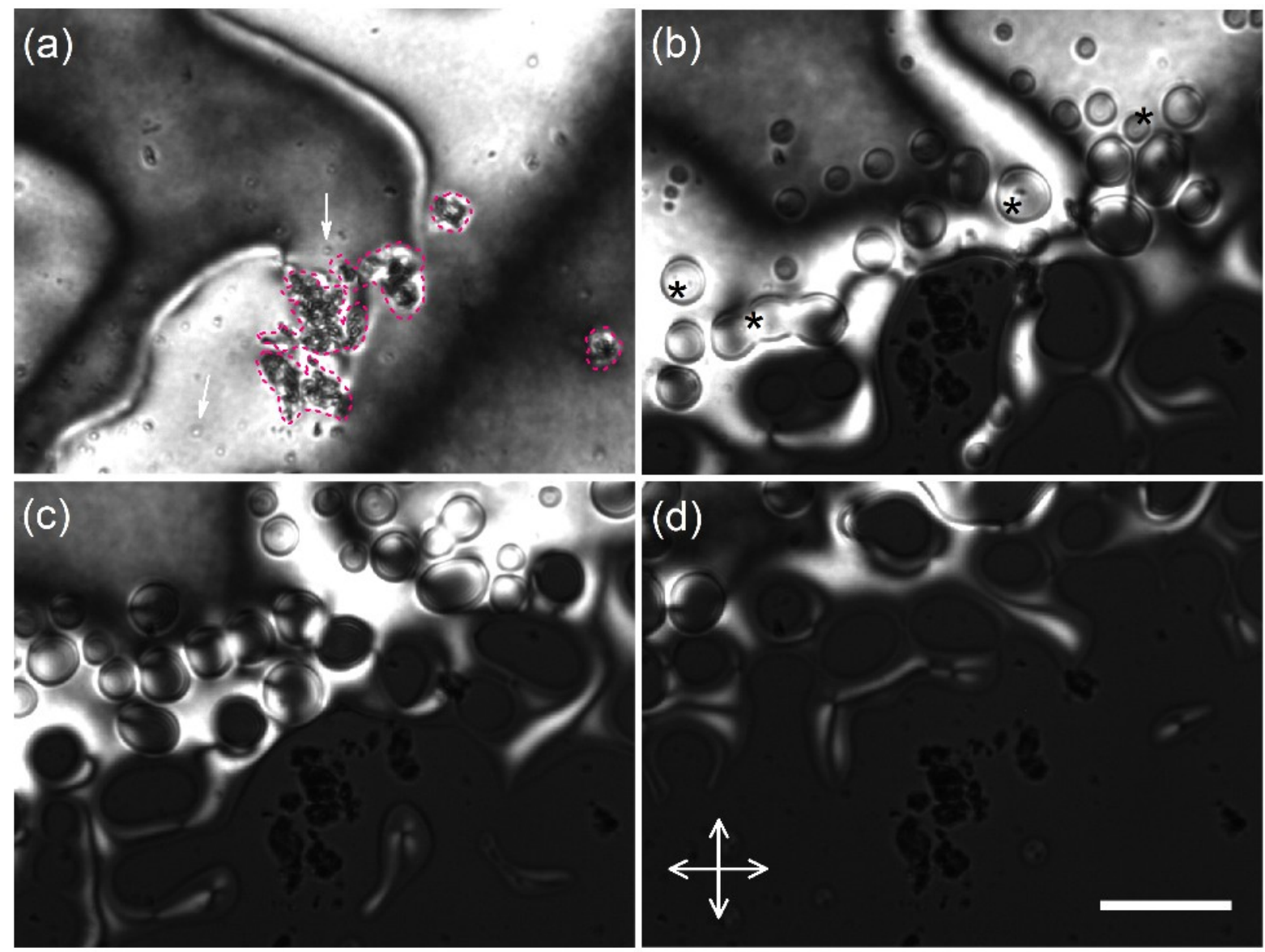

Fig. 4. Monitoring the $\mathrm{N}-\mathrm{I}$ phase transition of the $5 \mathrm{CB}-\mathrm{IONP}$ mixture (“IONP-rich", $7 \times 10^{15} / \mathrm{mL}$ ) by using polarizing microscopy. (a) A complete nematic phase $\left(T=32.0{ }^{\circ} \mathrm{C}\right.$ ) where the localized granules (dashed lines) are clustered and located at (or near to) the defect sites. The isolated granules are seen in the nematic matrix (arrows). (b) Local melting begins from the granules shown in (a), which are indicated by *. (c-d) The melted domains grow, coalesce, and become larger isotropic areas with time. The time interval between each adjacent image is 2-3 s, and the total increase in temperature from (a) to (d) is less than $0.1{ }^{\circ} \mathrm{C}$. Scale bar, $50 \mu \mathrm{m}$.

\section{Conclusion}

In this study, we observed structures of IONP aggregates in a host nematic 5CB liquid crystal by cryoTEM and investigated their role in the $\mathrm{N}-\mathrm{I}$ phase transition using polarized light microscopy.

In the nematic matrix, the dopant IONPs (with an average diameter of $5.8 \pm 1.2 \mathrm{~nm}$ for each IONP) appeared to be packed in roughly spherical aggregates. These aggregates have a diameter of $35 \pm 9$ $\mathrm{nm}$ and consist of several tens of IONPs. The spacing between the IONPs in the aggregates is $7.7 \pm 1.0 \mathrm{~nm}$, which is $2 \mathrm{~nm}$ larger than their intrinsic diameter. This $2 \mathrm{~nm}$ separation can be explained by the length of oleic acid chains which are coated on the IONP surface.

The IONP aggregates are clustered in an elongated morphology with the length of a few hundreds of $\mathrm{nm}$ and are, therefore, visualized as a point object in conventional optical microscopy. In IONP-doped $5 \mathrm{CB}$, we indeed observed granules, via polarized light microscopy, with an average diameter of $2.0 \pm 0.6 \mu \mathrm{m}$. The granules (i.e., scattering centers) are never observed in pure 5CB without IONPs and the number of granules increased with an increasing concentration of IONPs. The granules are 
primarily located at the defect sites in the nematic phase, and act as nucleation centers for the growth of isotropic domains during the $\mathrm{N}-\mathrm{I}$ phase transition.

\section{Acknowledgement}

We thank Dr. Min Gao for his technical help using the cryo-TEM facility at the Liquid Crystal Institute (Kent State University), supported by the Ohio Research Scholars Program "Research Cluster on Surfaces in Advanced Materials." We also would like to thank Dr. Min-Ho Kim (Biological Sciences, Kent State University) for his advice and fruitful discussions. This work was partly supported by the Nanomaterial Technology Development Program (NRF-2017M3A7B6052455) funded by the South Korean Ministry of Science and ICT.

\section{References}

[1] Y. Shiraishi, N. Toshima, K. Maeda, H. Yoshikawa, J. Xu, S. Kobayashi, Appl. Phys. Lett. 2002, 81, 2845.

[2] P. Nayek, G. Li, Sci. Rep. 2015, 5, 10845.

[3] N. Podoliak, D. Bartczak, O. Buchnev, A. G. Kanaras, M. Kaczmarek, J. Phys. Chem. C 2012, 116, 12934.

[4] E. S. Iranizad, Z. Dehgani, M. Nadafan, J. Mol. Liq. 2014, 190, 6.

[5] X. Li, C. Yang, Q. Wang, D. Jia, L. Hu, Z. Peng, L. Xuan, Opt. Commun. 2013, 286, 224.

[6] S. Kobayashi, T. Miyama, N. Nishida, Y. Sakai, H. Shiraki, Y. Shiraishi, N. Toshima, J. Display Technol. 2006, 2, 121.

[7] J. S. Roy, T. P. Majumder, R. Dąbrowski, Funct. Mater. Lett. 2014, 7, 1450054.

[8] A. Mouhli, H. Ayeb, T. Othman, J. Fresnais, V. Dupuis, I. R. Nemitz, J. S. Pendery, C. Rosenblatt, O. Sandre, E. Lacaze, Phys. Rev. E 2017, 96, 012706.

[9] I. Appel, H. Nádasi, C. Reitz, N. Sebastián, H. Hahn, A. Eremin, R. Stannarius, S. S. Behrens, Phys. Chem. Chem. Phys. 2017, 19, 12127.

[10] T. Hegmann, H. Qi, V. M. Marx, J. Inorg. Organometal. Polym. Mater. 2007, 17, 483-508.

[11] C. N. Melton, S. T. Riahinasab, A. Keshavarz, B. J. Stokes, L. S. Hirst, Nanomaterials 2018, 8, 146.

[12] C. Da Cruz, O. Sandre, V. Cabuil, J. Phys. Chem. B 2005, 109, 14292.

[13] A. L. Rodarte, R. J. Pandolfi, S. Ghost, L. S. Hirst, J. Mater. Chem. C 2013, 1, 5527-5532.

[14] R. Basu, G. S. lannacchione, Phys. Rev. E 2009, 80, 010701.

[15] X. Wang, D. S. Miller, E. Bukusglu, J. J. de Pablo, N. L. Abbott, Nat. Mater. 2016, 15, 106.

[16] P. J. Collings, M. Hird, Introduction to Liquid Crystals: Chemistry and Physics, Taylor \& Francis, London, UK 1997.

[17] R. Basu, Phys. Rev. E 2014, 89, 022508.

[18] S. Dutz, R. Hergt, Int. J. Hyperthermia 2013, 29, 790.

[19] H. Yan, B. Sung, M-H. Kim, C. Kim, Mater. Res. Express 2014, 1, 045032.

[20] M. Gao, Y-K. Kim, C. Zhang, V. Borshch, S. Zhou, H-S. Park, A. Jákli, O. D. Lavrentovich, M-G. Tamba, A. Kohlmeier, G. H. Mehl, W. Weissflog, D. Studer, B. Zuber, H. Gnägi, F. Lin, Microsc. Res. Tech. 2014, 77, 754.

[21] B. Sung, A. Leforestier, F. Livolant, Nucleic Acids Res. 2016, 44, 1421.

[22] E. R. Garland, E. P. Rosen, L. I. Clarke, T. Baer, Phys. Chem. Chem. Phys. 2008, 10, 3156.

[23] P. Lesiak, K. Bednarska, W. Lewandowski, M. Wójcik, S. Polakiewicz, M. Bagiński, T. Osuch, K. Markowski, K. Orzechowski, M. Makowski, J. Bolek, T. R. Woliński, ACS Nano 2019, 13, 10154.

[24] H. Yoshida, K. Kawamoto, H. Kubo, T. Tsuda, A. Fujii, S. Kuwabata, M. Ozaki, Adv. Mater. 2010, $22,622$.

[25] S-J. Hwang, S-C. Jeng, C-Y. Yang, C-W. Kuo, C-C. Liao, J. Phys. D: Appl. Phys. 2009, 42, 025102.

[26] A. N. G. Parra-Vasquez, L. Oudjedi, L. Cognet, B. Lounis, J. Phys. Chem. Lett. 2012, 3, 1400-1403.

[27] A. C. Rauch, S. Garg, D. T. Jacobs, J. Chem. Phys. 2002, 116, 2213-2218. 


\section{Highlights}

- Cryo-TEM shows how metallic nanoparticles are dispersed in a liquid crystal matrix.

- Morphology and structure of nanoparticle aggregates are visualized in situ.

- Nanoparticle aggregates affect the patterns of nematic-isotropic phase transition.

\section{Graphical Abstract}
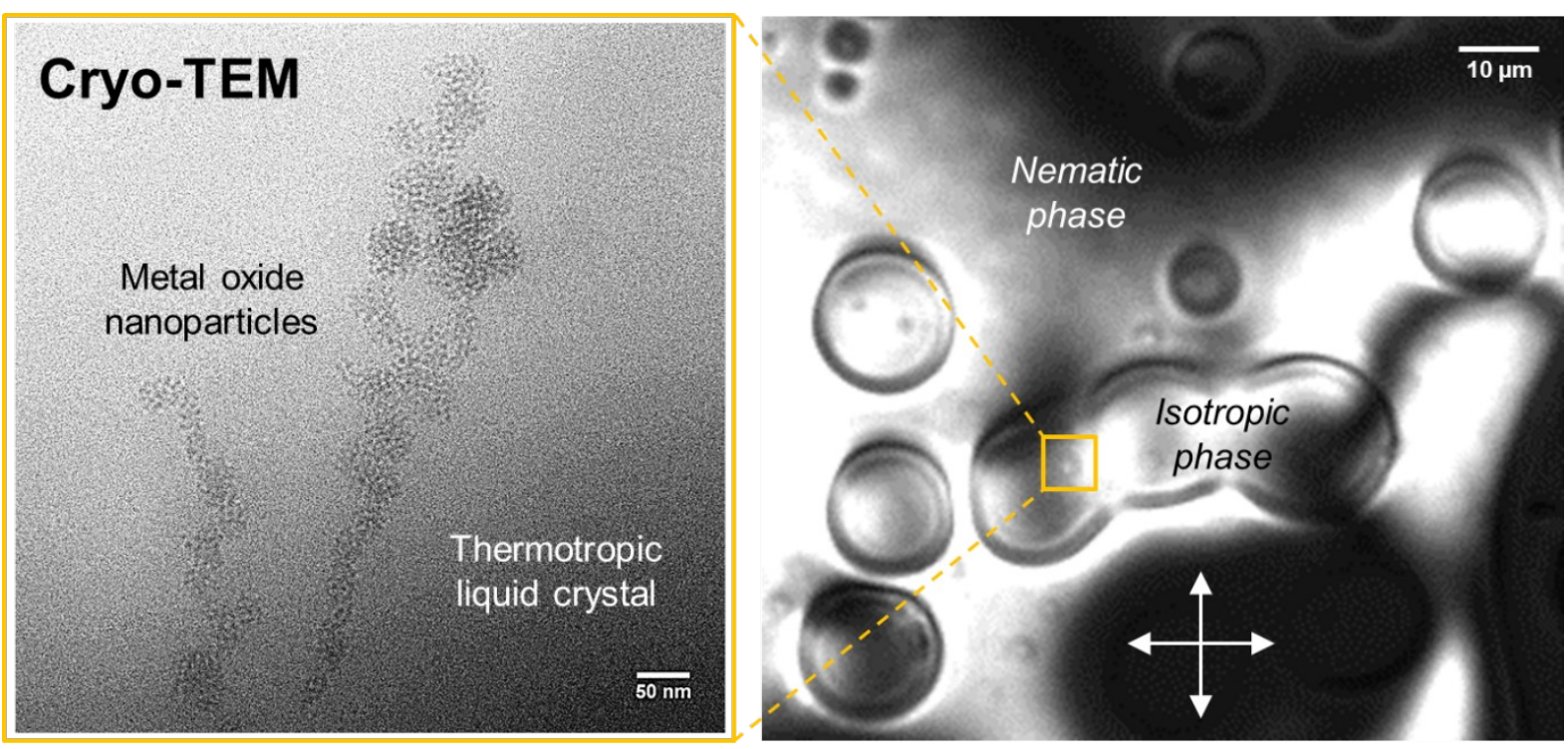